\title{
Index autorum ad Vol. 155
}

\section{Confecit: C. Loeb-SchÜГch}

(B) $=$ Buchbesp $\Gamma$ echungen - Book reviews - Livres nouveaux $(V)=$ Vortrag - Report -

Communication

Agoston, I., 399

Alkemade, P. P. H., 70 (V), 317 (V)

Alpern, M., 63 (V)

Arseni, C, 379

Asayama, R., 117

Auerbach, E., 98

Bacskulin, J., 433 Balthasar, K., 249 Behnke, H., 81 Berghoffer, B., 205 Berghuis, G., 313 (V)

Bettman, J. W., 427 (B) Bonnet, R., 429 (B) Bouman, M. A., 429 (B)

Cagianut, B., 148 Carp, N., 379 Castrén, J. A., 194 Castroviejo, R., 429 (B) Crone, R. A., 37 (V)

Cuendet, J. F., 186

David, M., 428 (B) Dekking, H. M. †, 332 (V) Delleman, J. W., 409 Dilenge, D., 428 (B)

Doeschate, J. ten, v. Ten

Doeschate, J. Dollmann, A., 390 Donaldson, D., 430 (B) Donders, P. C, 308 (V)

Fischgold, H., 428 (B) Follmann, P., 433 Francois, J., 169, 337, 433

Gansner, Johanna, 234 Garipuy, J., 217 Gloor, B., 449 Gobin, M. H., 297 (V) Gomperts, C. E., 130 Grieten, J., 1 Gróf, P., 399

Hanssens, M., 169, 337 Harris, J. E., 428 (B) Heimann, K., 390 Hemmes, G. D., 57 (V), 300 (V)

Henkes, H.E., 17 (V), 28 (V) Hoffmann, D. H., 476 (B) Hoster, F. A., 477 (B)

Jaeger, W., 390 Junge, J., 291 (V)

Karel, I., 8 Karjalainen, K., 194 Kelmann, C. D., 430 (B) King, J. H., 427 (B) Küchle, H. J., 427 (B)

Lalive d'Epinay-Zimmerli, Ruth, 271

Lennes, G., 1

Lerman, S., 476 (B)

Lith, G. H. M., van, v. Van Lith,

G. H. M. Luparello, Th. J., 430 (B)

Manschot, W. A., 66 (V) , 294 (V) Maretsis, M., 379 McTigue, J. W., 427 (B) Monacelli, M., 477 (B) Mulock Houwer, A. W., 67 (V)

Nazzaro, P., 477 (B)

Oda, I., 464

Oosterhuis, J. A., 36 (V), 357

O'Rourke, J., 205

Pakarinen, P., 194 Pigassou, Renée, 217 Pohjola, S., 194

Randolph, 477 (B) Rowe, H.. 98

488

Index autorum ad Vol. 155 
Schreinemachers, H. P., 17 (V)

Schulze, F., 87

Schweitzer, N. M. J., 80 (V), 299 (V)

Selisko, G., 87

Sévin, R., 186

Stockmans, L., 337

Ten Doeschate, J., 63 (V)

Ten Thye, P. A. †, 77 (V), 333 (V)

Thiel, H. J., 81

Tost, M., 87

Troelstra, A., 80 (V)

Tsukahara, I., 117

Van Lith, G. H. M., 78 (V) Vasilescu, Alexandra, 379 Vassura, G. W., 128 (B) Verduin, P. C., 28 (V) Victoria-Troncoso, V., 169 Vos, J. J., 429 (B)

Weekers, R., 1 Winkelman, J. E. , 409 Worst, J. G. F., 56 (V),330 (V)

Y. Yoshida

E.

117

Zagora

E.

141 